\title{
ANÁLISE DE FILMES DE ESCÓRIA DE FLUXANTES APLICADOS EM AÇOS BAIXO E MÉDIO CARBONO*
}

\author{
Alexandra de Almeida Diego ${ }^{1}$ \\ Cláudio Antônio Goulart Valadares ${ }^{2}$ \\ Carolina Campelo Bezerra ${ }^{3}$ \\ Leonardo Martins Demuner ${ }^{4}$ \\ Gabriel Romeu Trindade dos Santo ${ }^{5}$ \\ Rodrigo Duda ${ }^{6}$ \\ Fernando Vernilli Junior
}

\section{Resumo}

O controle da transferência de calor é uma das principais funções que o fluxante exerce durante aplicação no molde de lingotamento contínuo. O fluxo de calor na interface placa / molde deve estar adequado aos parâmetros de processo e ao tipo de aço, caso contrário pode provocar preferencialmente alarmes de agarramento em aços baixo carbono e trincas longitudinais em aços médio carbono, podendo levar até ao breakout. Este trabalho tem o objetivo de caracterizar os filmes de escória aplicados em aços baixo (Filme BC) e médio carbono (Filme MC), ambos comerciais, utilizando as análises de Microscopia Eletrônica de Varredura (MEV) com Energia Dispersiva de raios-X (EDS) acoplado e Difratometria de raios-X (DRX). As diferenças entre os filmes de escórias foram discutidas e relacionadas aos dados presentes na literatura.

Palavras-chave: Transferência de calor; Fluxante; Cuspidina; Lingotamento contínuo.

\section{ANALYSIS OF SLAG FILMS APPLIED IN LOW AND MEDIUM CARBON STEEL Abstract}

The heat transfer control is one of the main function that the mould flux works during the application in the mold at the continuous casting machine. The heat flux at the interface mold / strand must be appropriate to the process parameters and type of steel, otherwise it may cause preferably alarms in low carbon steels and longitudinal cracks in medium carbon steels, which may lead to the breakout. This work aims to characterize the slag films applied in low and medium carbon steels, both commercial, using the Scanning Electron Microscopy (SEM) with Energy Dispersive Scanning (EDS) coupled and X-ray Diffraction (XRD). The differences between then were discussed and compared with data from literature.

Keywords: Heat transfer; Mould flux; Cuspidine; Continuous casting.

1 Engenheira de Materiais, Mestranda em Engenharia de Materiais, EEL-USP, Engenheira de Desenvolvimento de Produto, Carboox Resende Química, Resende, RJ, Brasil.

2 M.Sc, CQE/ASQ, Engenheiro Metalurgista, Diretor Técnico, Carboox Resende Química Resende, RJ, Brasil.

3 Mestre em Engenharia, Engenheira de Produção Química, Vice Presidente, Carboox Resende Química, Resende, RJ, Brasil.

4 Engenheiro de Produção, MBA em gerenciamento de projetos, Gerente de Lingotamento Contínuo, ThyssenKrupp CSA, Itaguaí, RJ, Brasil.

5 Engenheiro Metalurgista, Eng. Metalúrgico Jr, ThyssenKrupp CSA, Itaguaí, RJ, Brasil.

6 Tecnólogo em Metalurgia e Materiais, Assistente Técnico, Carboox Resende Química, Resende, RJ, Brasil.

7 Eng. Químico, Ph.D., Coordenador do Programa de Pós-Graduação em Engenharia de Materiais, EEL-USP, Lorena, SP, Brasil. 


\section{INTRODUÇÃO}

O fluxante é uma mistura de materiais não metálicos que em contato com o aço líquido, funde e exerce cinco funções, conforme descrito na Figura 1. Na superfície do aço líquido tem as funções de isolar termicamente, proteger o aço contra a reoxidação e absorver inclusões principalmente $\mathrm{Al}_{2} \mathrm{O}_{3}$. Na interface entre molde e a pele de aço em solidificação atua como lubrificante e no controle do fluxo de calor.

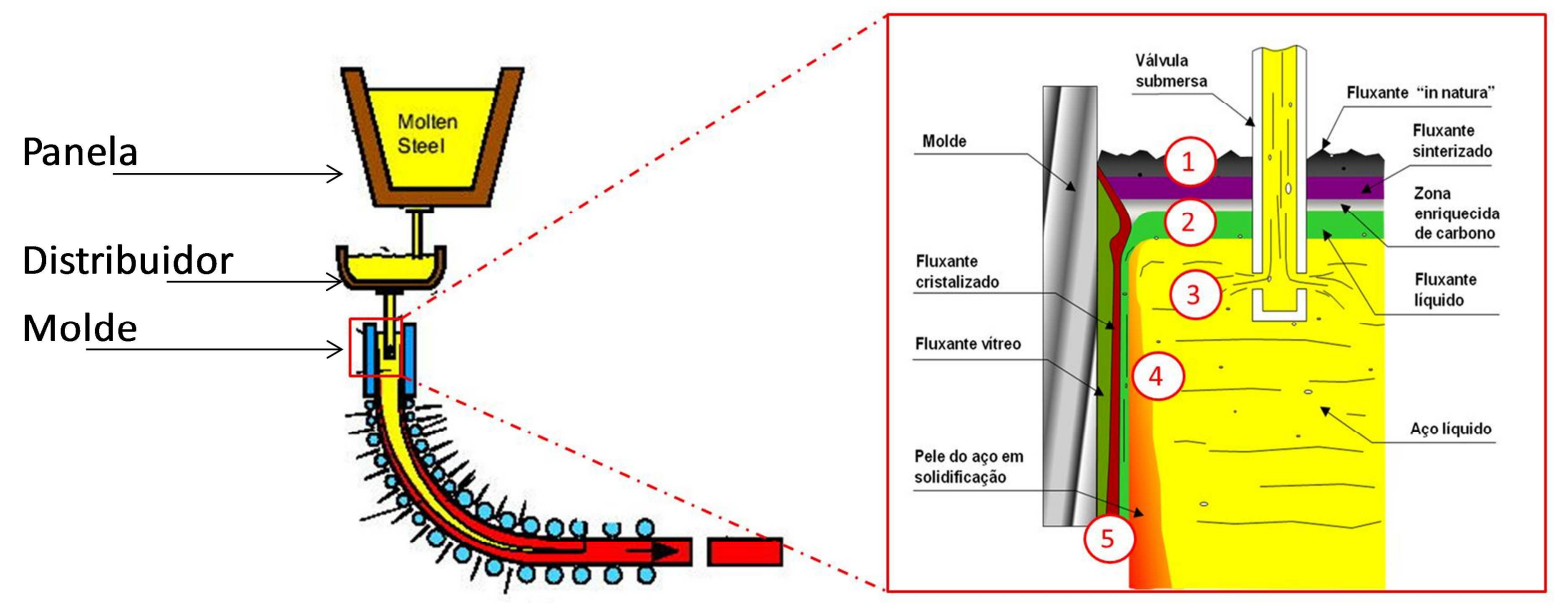

(a)

(b)

Figura 1. (a) Diagrama esquemático do processo de lingotamento contínuo. (b) Mostra as camadas que o fluxante forma sobre o aço líquido e na interface entre o molde e a pele de aço em solidificação com as funções enumeradas de 1 a 5, sendo: 1 Isolamento Térmico; 2 Prevenção da Reoxidação; 3 Absorção de Inclusões; 4 Lubrificação e 5 Controle da Transferência de Calor (adaptado de [1]).

O controle da transferência de calor, identificada com o número 5 na Figura $1 \mathrm{~b}$, tem influência direta na qualidade superficial das placas de aço. Em especial em aços médio carbono, devido a contração térmica de aproximadamente $4 \%$ decorrente da transformação da fase $\delta$-ferrita em $\mathrm{y}$-austenita durante 0 resfriamento, provocando tensões que aliviadas com podem gerar trincas. Como solução para este problema, trabalha-se com a pele de aço em solidificação fina e uniforme, através da menor extração de calor na interface entre o veio e o molde. Neste caso, é necessário que o filme de escória que se forma entre o veio e o molde seja mais espesso [2]. Isso é possível com o aumento da basicidade e da temperatura de cristalização do fluxante, tendo como consequência o aumento da taxa de cristalização [3].

A temperatura de cristalização é o início da formação de cristais no filme de fluxante fundido entre o aço e o molde, alguns estudos mostram sua influencia na transferência de calor [4-5] e o seu aumento com a basicidade [6]. A taxa de cristalização do filme de escória sólido é o principal item que controla a transferência de calor, pois aumenta a resistência térmica, suavizando a extração de calor. Muitos estudos citam a fase Cuspidina $\left(\mathrm{F}_{2} \mathrm{Ca}_{4} \mathrm{Si}_{2} \mathrm{O}_{7}\right)$ como principal fase cristalina formada no filme de escória sólido [7-16].

Os mecanismos envolvidos no processo de transferência de calor consistem basicamente na condução e radiação [17], conforme ilustrado na Figura 2. A condução térmica é a principal responsável pela transferência de calor e a contribuição da radiação é cerca de $20 \%$ nas camadas de escórias líquida e cristalina. A camada cristalina reduz com eficiência o fluxo térmico, pois os cristais sólidos difratam a radiação [18-19]. 


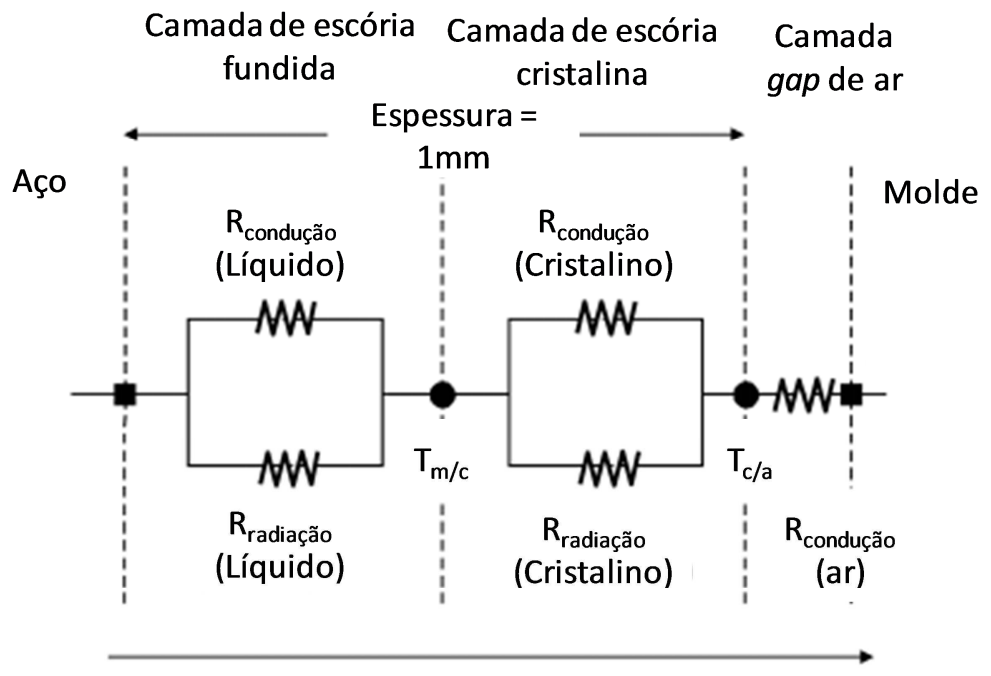

fluxo de condução térmica total

Figura 2. Modelo de fluxo térmico entre a pele de aço e o molde, onde $R=$ resistência para condução térmica, $\mathrm{T}_{\mathrm{m} / \mathrm{c}}=$ temperatura da interface escória líquida / cristalina e $\mathrm{T}_{\mathrm{c} / \mathrm{a}}=$ temperatura da interface escória cristalina / gap de ar (adaptado de [18]).

Este trabalho tem o objetivo de avaliar através das análises de MEV, EDS e DRX as amostras de filmes de escória coletados entre a placa e o molde durante 0 lingotamento contínuo de aços baixo e médio carbono. As diferenças entre eles foram discutidas e comparadas com a literatura.

\section{MATERIAIS E MÉTODOS}

Para este trabalho foram estudados 2 (dois) fluxantes com diferentes composições químicas e consequentemente temperatura de cristalização diferentes, Tabela 1. Na preparação de amostra para análise do MEV, ambos os filmes de escória foram preparados com o objetivo de visualizar a região da interface entre o molde e a placa. As amostras foram preparadas através do embutimento a frio com resina de cura rápida, evitando assim tensões externas na amostra. A operação de desbaste foi conduzida com lixas de $\mathrm{SiC}$ comerciais de 500 a 2400 "mesh". O polimento foi executado com pano de polimento e suspensão de sílica coloidal 0,05 $\mu \mathrm{m}$. O MEV foi realizado com o objetivo de avaliar em maior resolução a interface entre placa e molde através das diferenças de tons de cinza que indicam diferenças de composição química e topografia. O MEV utilizado foi o Hitachi 3000 com EDS acoplado. O uso do EDS teve como objetivo avaliar qualitativamente a variação dos elementos químicos ao longo da interface do filme de escória entre placa e molde.

Para a análise via DRX, as amostras foram moídas no moinho de bolas até obtido $100 \%$ passante na peneira \#325. O DRX foi aplicado com o objetivo de determinar as fases mineralógicas constituintes, determinadas através da consulta de um banco de dados contendo informações cristalográficas básicas de compostos cristalinos. Foi utilizado o difratômetro modelo Empyrian da Panalytical com o passo angular de $0,013^{\circ}$, tempo de leitura por passo de 30 s e na faixa angular de 10 a $90^{\circ}$.

\section{RESULTADOS E DISCUSSÃO}

Os filmes de escória são originalmente de fluxantes com a composição química e temperatura de cristalização descritos na Tabela 1. 
Tabela 1. Composição química em \% dos fluxantes BC (baixo carbono) e fluxante MC (médio carbono) e temperatura de cristalização.

\begin{tabular}{cccccccc} 
& $\mathrm{SiO}_{2}$ & $\mathbf{C a O}$ & $\mathrm{Al}_{2} \mathrm{O}_{3}$ & $\mathbf{N a}_{2} \mathbf{O}$ & $\mathbf{F}$ & Basicidade & Temp. Cristalização $\left({ }^{\circ} \mathbf{C}\right)$ \\
\hline Fluxante BC & 34,0 & 34,5 & 4,5 & 7,5 & 6,0 & 1,02 & 1142 \\
\hline Fluxante MC & 31,5 & 36,0 & 4,5 & 6,5 & 10,5 & 1,15 & 1186
\end{tabular}

As imagens obtidas por MEV, são mostradas nas Figuras 3a e 3b. Ambos são da região do menisco, mostrando a quina do molde e a interface entre placa e molde. $\mathrm{O}$ lado do molde é a região superior e o lado da placa é a região inferior de ambas as figuras.

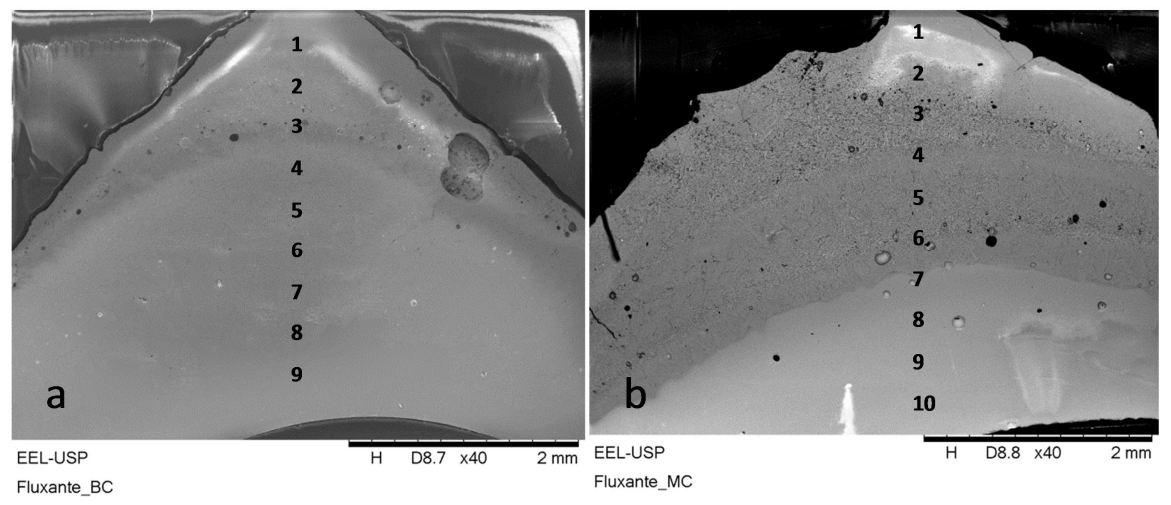

Figura 3. Filmes de escória solidificados retirados da interface entre molde e placa, da quina e região do menisco. A parte superior é o lado do molde e a parte inferior é o lado da placa e ambos apresenta ampliação de 40 vezes a) Filme BC, valores de 1 a 9 das análises elementares EDS e b) Filme MC, valores 1 a 10 das análises elementares EDS.

$\mathrm{Na}$ Figura 3a, a imagem do Filme BC ao longo da interface placa-molde é mais homogênea comparado ao Filme MC. Este fato pode estar relacionado a composição química, Tabela 1, pois o Filme BC tem menor basicidade e menor temperatura de cristalização que forma uma escória com menor tendência a formação de cristais, fato coerente com as imagens mostradas na Figura $3 \mathrm{a}$. $\mathrm{Na}$ figura $3 b$ o Filme MC apresenta regiões mais distintas, com a formação mais pronunciada de dendritas até uma região mais homogênea. Segue abaixo a Figura 4 mostrando com maior resolução regiões distintas no Filme MC. Na Figura 4 a maior ampliação evidencia esta observação de modo que podemos relacionar naturalmente com os resultados de DRX que acusam a presença da fase cristalina Cuspidina, Figura 5 e 6. 


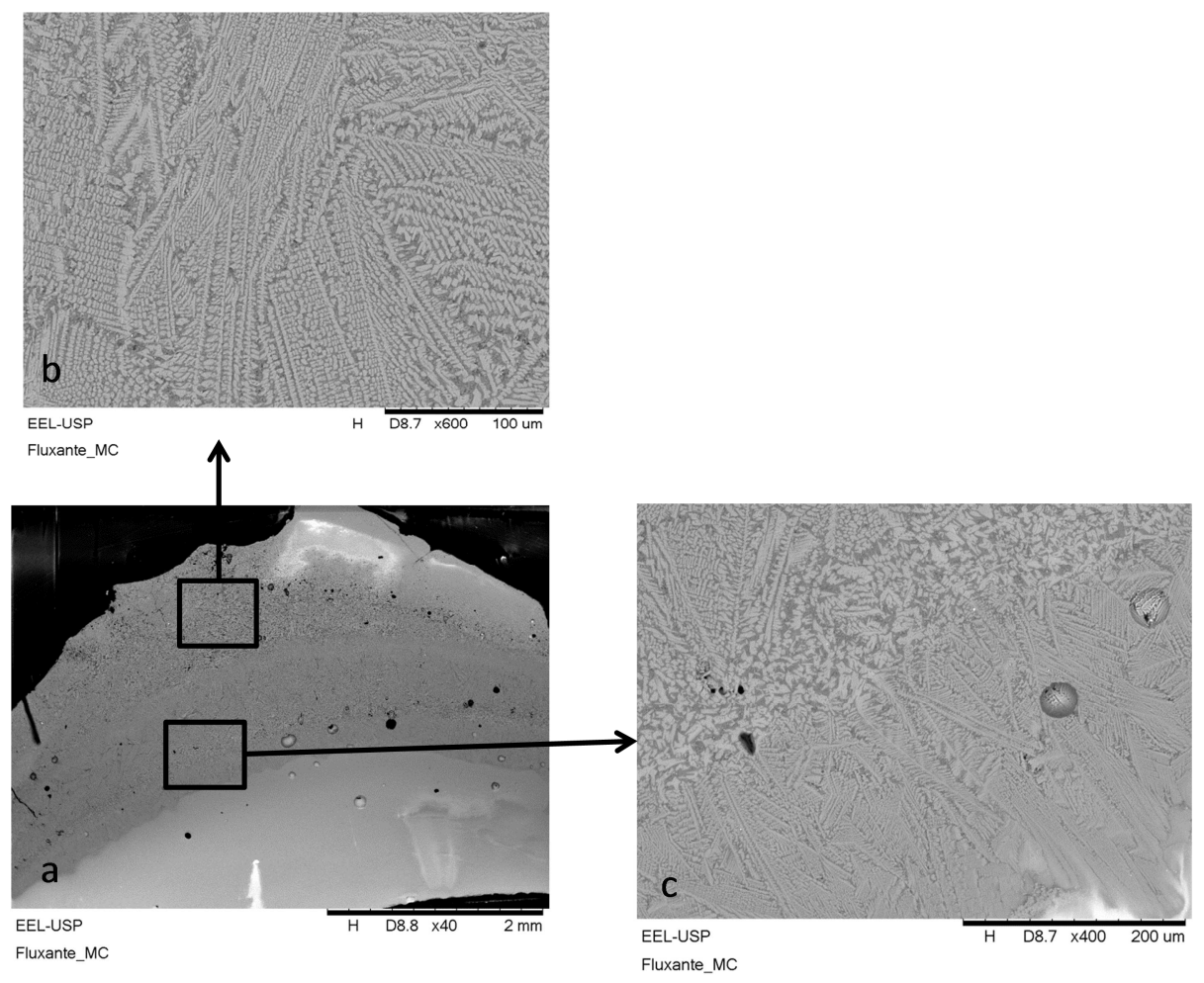

Figura 4. a) Filme MC destacando as diferenças de morfologia ao longo da interface, b) detalhe na região próxima ao molde mostrando dendritas e c) detalhe da região do meio do filme mostrando a região intermediária que de um lado mostra dendritas até uma região mais homogênea.

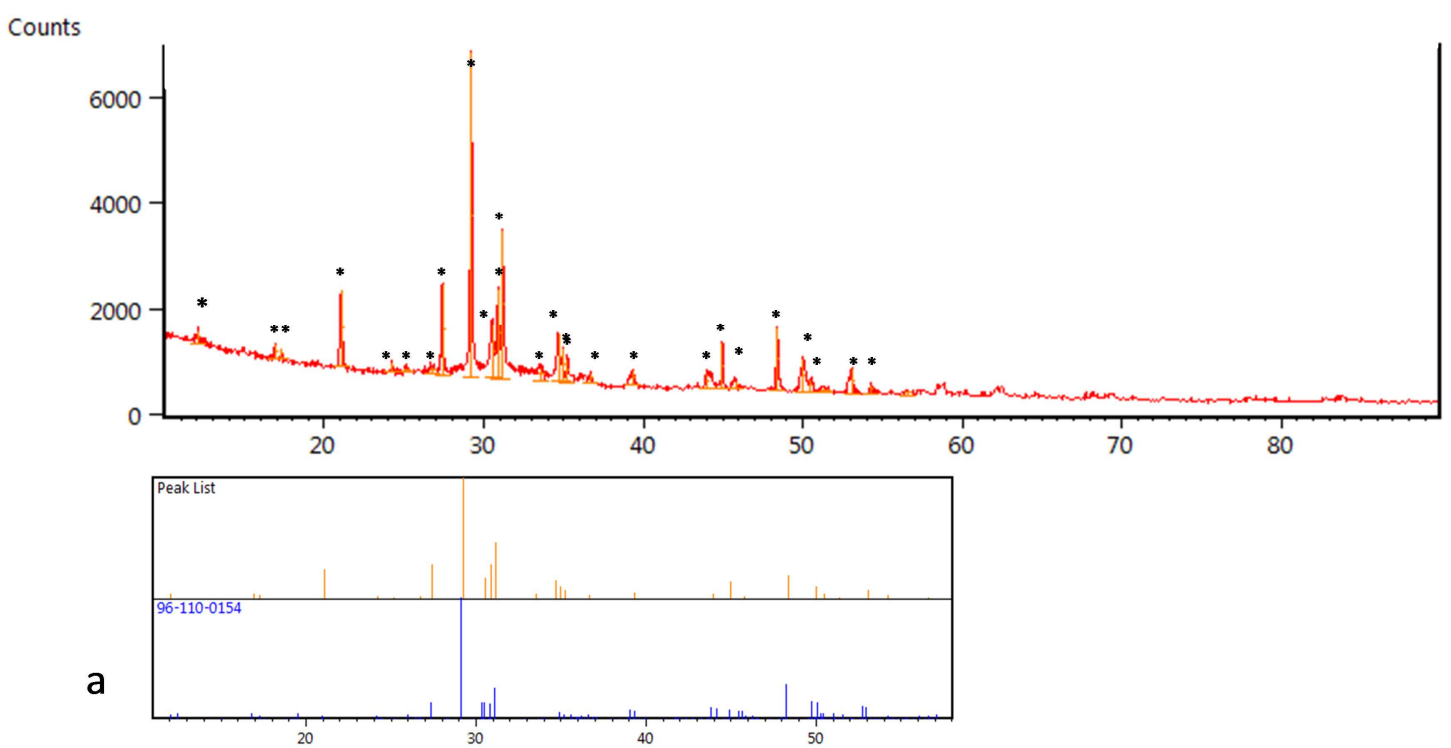

Figura 5. DRX do Filme BC de escória com 33\% de probabilidade da fase Cuspidina. 
Counts
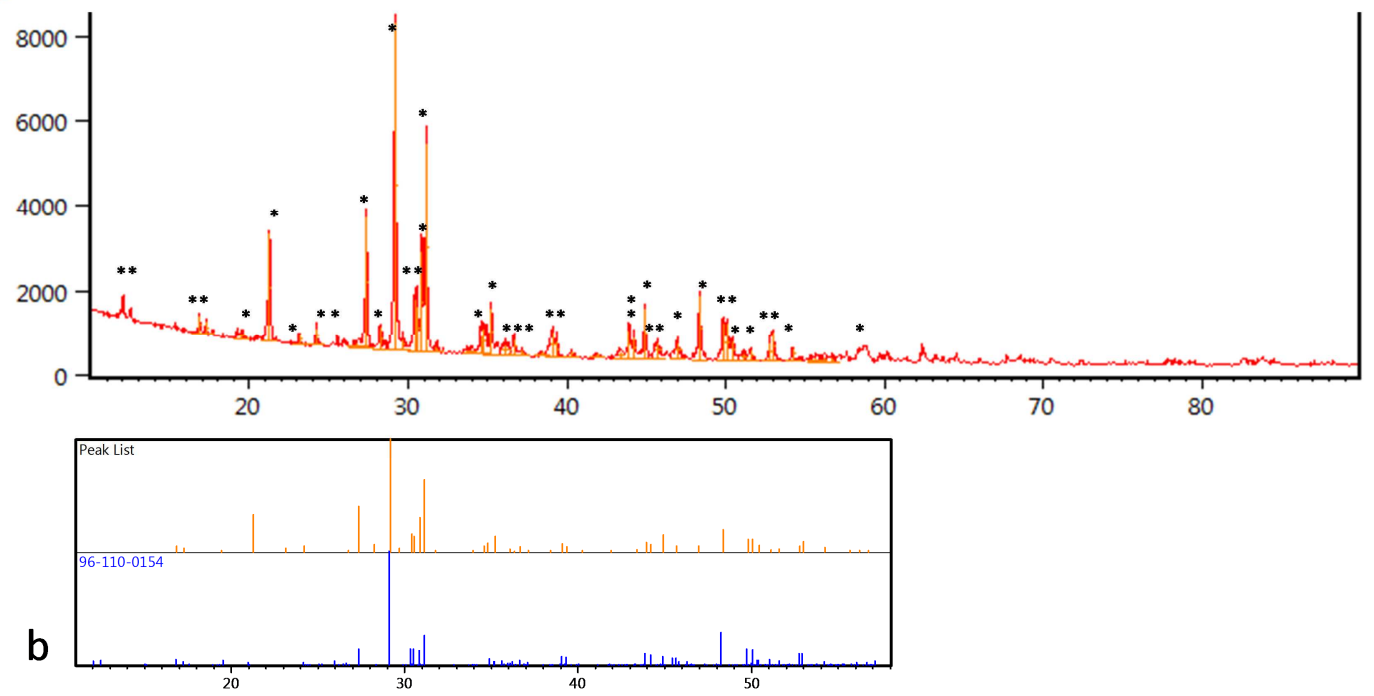

Figura 6. DRX do Filmes MC com 64\% de probabilidade da fase Cuspidina.

Figura 4c, a ampliação na região intermediária foi de 400 vezes e próxima ao molde, Figura 4b foi de 600 vezes. É possível ver com detalhes as alterações nos tons de cinza de claro a escuro indicando diferença na composição quimica. Este fato pode ser relacionado a segregação comum na formação de cristais, observado com a formação de dendritas e regiões distintas ao longo do molde. As diferenças visuais na microestrutura do interior e da região próxima ao molde podem ter ocorrido devido a diferenças na taxa de resfriamento, até porque o intervalo de temperatura do molde a placa pode chegar a $1000^{\circ} \mathrm{C}$, variando de aproximadamente $200^{\circ} \mathrm{C}$ até $1200^{\circ} \mathrm{C}$.

A Figura 7 mostra os resultados das análises pontuais qualitativas obtidos no MEVEDS ao longo dos filmes de escória, conforme indicação na Figura 3a e 3b.

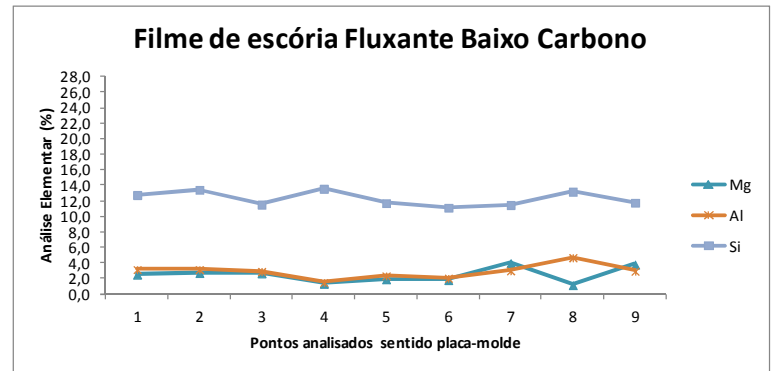

a) Resultados EDS fluxante BC elementos $\mathrm{Mg}$, Al e Si

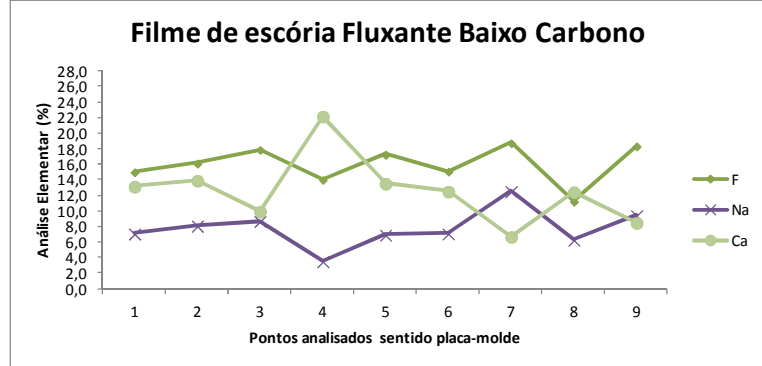

c) Resultados EDS fluxante BC elementos $F$, $\mathrm{Na}, \mathrm{Ca}$

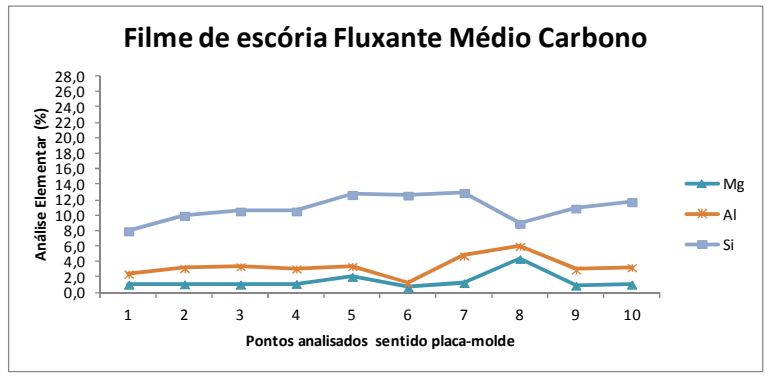

b) Resultados EDS fluxante MC elementos $\mathrm{Mg}$, Al e Si

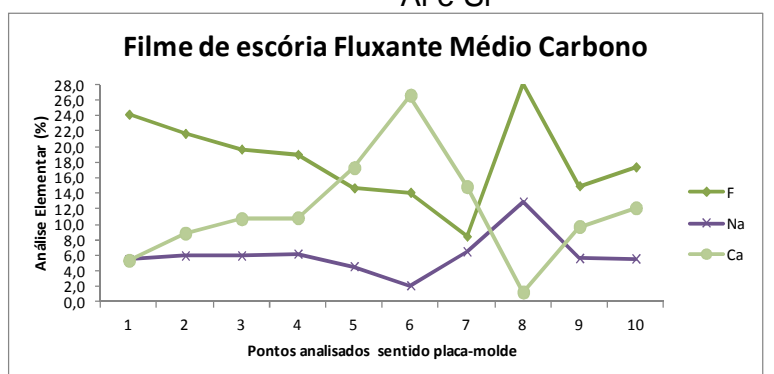

d) Resultados EDS fluxante MC elementos $F$, $\mathrm{Na}, \mathrm{Ca}$

Figura 7. Resultados qualitativos das análises pontuais via EDS realizadas na interface e sentido placa-molde, conforme números sequenciados indicados nas Figuras $4 \mathrm{a}$ e $4 \mathrm{~b}$. Os resultados mostram a tendência de variação dos elementos ao longo da interface. 
A Figura 7 mostra os resultados das análises pontuais obtidas no MEV-EDS realizadas na interface e sentido placa-molde em ambos os filmes, BC e MC. Os elementos que mostraram a tendencia de menor variação ao longo do Filme $\mathrm{BC}$ e $\mathrm{MC}$ foram $\mathrm{Mg}, \mathrm{Al}$ e Si. Já os elementos $\mathrm{F}, \mathrm{Na}$ e $\mathrm{Ca}$ apresentaram a tendencia de maior variação ao longo dos filmes. Este fato é mais pronunciado no Filme MC, que pode ser relacionado a maior formação de dendritas observadas na imagens do MEV, Figura 4. A maior variação dos elementos $\mathrm{Ca}$ e $\mathrm{F}$ é provavelmente devido a segregação que ocorre durante a formação da fase Cuspidina, demonstrada no resultado de DRX Figura 5 e 6.

A menor formação de cristais no Filme BC pode ser relacionado a menor resistência ao fluxo térmico por radiação. Esta característica é adequada aos aços baixo carbono que permitem maior fluxo térmico da placa para o molde, são lingotados com maior espessura de pele de aço com o objetivo de reduzir as chances de agarramento no molde, fato também encontrado em outros estudos na literatura [5-6,20-21]. A presença de fase cristalina no Filme MC está coerente com estudos que citam que a maior taxa de cristalização aumenta o controle da transferência térmica no sentido de suavizar o fluxo de calor e consequente redução da espessura da pele de aço visando reduzir as chances de ocorrência de trincas longitudinais $[5,13]$ devido a difração da radiação nos cristais.

\section{CONCLUSÃO}

- A maior formação de cristais ocorrida no Filme MC pode ser relacionado a composição química, sabendo que apresenta maiores valores de basicidade e temperatura de cristalização comparados ao Filme BC, sendo assim mais favorável a formação da estrutura com dendritas conforme imagens obtidas por MEV;

- As imagens obtidas do Filme BC também podem ser relacionadas a composição química, pois sabe-se que a basicidade e a temperatura de cristalização são menores, então existe a tendência de reprimir a formação de uma estrutura definida por dendritas;

- Foi identificado na análise por DRX que a fase cristalina formada nos filmes se trata da Cuspidina $\left(\mathrm{F}_{2} \mathrm{Ca}_{4} \mathrm{Si}_{2} \mathrm{O}_{7}\right)$ que é conhecida como principal barreira na condução térmica por radiação contribuindo para o controle da transferencia de calor na interface placa-molde;

- Os dados coletados no EDS, mostram que no filme MC apresentou maior variação dos elementos $\mathrm{Ca}$ e $\mathrm{F}$ ao longo do filme de escória, possivelmente devido a segregação ocorrida durante a formação de cristais;

- A variação dos elementos $\mathrm{Ca}$ e $\mathrm{F}$ ao longo do Filme BC também foi observado mas com menor evidência provavelmente devido a menor tendência a formação de cristais, mas ainda existente devido a evidencia de no entanto a indexação da fase Cuspidina evidencia a presença destes dois elementos.

\section{Agradecimentos}

Os autores agradecem a Escola de Engenharia de Lorena EEL-USP pelo apoio na execução dos ensaios. 


\section{REFERÊNCIAS}

1 Mills KC, Fox AB, Thackray RP, Li Z. The performance and properties of mould fluxes. VII International Conference on Molten Slags Fluxes and Salts The South African Institute of Mining and Metalurgy, 2004: 713-721

2 Vermeulen Y, Divry E, Rigaud M. The influence of chemical composition on the crystallization and the heat transfer of synthetic mould fluxes. Canadian Metallurgical Quarterly, vol. 43, No 4, 2004: 527-534

3 Watanabe K, Suzuki M, Murakami K, Kondo H, Miyamoto A, Shiomi T. The Effect of Crystallization of Mold Powder on the Heat Transfer in Continuous Casting Mold. ISIJ, vol. 83 1997: 31-36

4 Orrling C, Cramb AW. The Effect of Water Vapor on Mold Slag Crystallization. Metallurgical and Materials Transactions B, vol 31B, April 2000: 403-406

5 Sridhar S, Mills KC, Afrange ODC, Lörz HP, Carli R. Break temperatures of mould fluxes and their relevance to Continuous Casting. Ironmaking and Steelmaking, vol. 27, No. 3, 2000: 238-242

6 Zhou L, Wanlin W, Fanjun M, Jin L, Juan W, Hiroyuki M, Fumitaka T. A Kinetic Study of the Effect of Basicity on the Mold Fluxes Crystallization. Metallurgical and Materials Transactions B, vol 43B, April, 2012: 354-362

7 Orrling C, Sridhar S, Cramb AW. In Situ Observation of the Role of Alumina Particles on the Crystallization Behavior of Slags. ISIJ International, vol. 40, No. 9, 2000: 877-885

8 Ryu HG, Zhang ZT, Cho J.W, Wen GH, Sridhar S. Crystallization Behaviors of Slags through a Heat Flux Simulator. ISIJ International, vol. 50, No. 8, 2010: 1142-1150

9 Watanabe T, Fukuyama H, Susa M. Phase diagram cuspidine (3CaO-2SiO2-CaF2)CaF2. Metallurgical and Materials Transactions B. 2000;31B:1273-1281

10 Watanabe T, Fukuyama H, Nagata K. Stability of Cuspidine (3CaO-2SiO2.CaF2) and Phase Relations in the CaO-SiO2-CaF2 System. ISIJ International. 2002;42(5):489-497

11 Nakada H, Nagata K. Crystallization of CaO-SiO2-TiO2 Slag as a Candidate for Fluorine Free Mold Flux. ISIJ International, vol. 46, No. 3, 2006: 441-449

12 Hanao M, Kawamoto M, Watanabe T. Influence of Na2O on Phase Relation between Mold Flux Composition and Cuspidine. ISIJ International, vol. 44, No. 5, 2004: 827-835

13 Hayashi M, Abas RA, Seetharaman S. Effect of crystallinity on thermal diffusivities of mould fluxes for the continuous casting of steels. ISIJ International. 2004;44(4):691-697

14 Meng Y, Thomas BG, Polycarpou AA, Prasad A, Henein H. Mould slag property measurements to characterize CC mold - shell gap phenomena. Canadian Metallurgical, vol 45, No 1, 2006: 79-94

15 Cruz A, Chávez F, Romero A, Palacios E, Arredondo V. Mineralogical phases formed by flux glasses in continuous casting mould. Journal of Materials Processing Technology. 2007;182:358-362

16 Mizuno H, Esaka H, Shinozuka K, Tamura M. Analysis of the Crystallization of Mold Flux for Continuous Casting of Steel. ISIJ International, vol. 48, No. 3, 2008: 277-285

17 Oliveira LJS, Lima MTD, Rodriguez FD, Valadares CAG, Diego AA. Avaliação da extração de calor no molde de lingotamento contínuo utilizando diferentes pós fluxantes. $42^{\circ}$ Seminário de Aciaria Internacional, 2011: 508-520

18 Nakada H, Susa M, Seko Y, Hayashi M, Nagata K. Mechanism of heat transfer reduction by crystallization of mold flux for continuous casting. ISIJ International. 2008;48(4):446-453

19 Santini L, Benavidez E, Brandaleze E. Evaluation of the mold fluxes crystallization by microscopy techniques. Acta Microscopica, vol. 19, No. 1, 2010: $1-8$

20 Valadares CAG, Diego AA, Bezerra MCC, et al. Análise de filmes de escória de fluxantes para aços baixo carbono. $45^{\circ}$ Seminário de Aciaria Internacional, Porto Alegre RS 2014

21 Seo M, Shi C, Cho J, et all. Crystallization behaviors of CaO-SiO2-Al2O3-Na2O-CaF2(Li2O-B2O3) mold fluxes. Metallurgical And Materials Transactions B, May 2014 